\title{
Preoperative Detection of Pleural Adhesions Using Ultrasonography for Ipsilateral Secondary Thoracic Surgery Patients
}

\author{
MOTOAKI YASUKAWA ${ }^{1}$, RYOSUKE TAIJI ${ }^{2}$, NAGAAKI MARUGAMI ${ }^{2}$, \\ TAKESHI KAWAGUCHI ${ }^{1}$, NORIYOSHI SAWABATA ${ }^{1}$, TAKASHI TOJO ${ }^{3}$, \\ JUNKO TAKAHAMA $^{2}$, NAOKI HAMAZAKI ${ }^{4}$, TOSHIKO HIRAI ${ }^{5}$ and SHIGEKI TANIGUCHI ${ }^{1}$ \\ ${ }^{1}$ Department of Thoracic and Cardiovascular Surgery, Nara Medical University School of Medicine, Nara, Japan; \\ ${ }^{2}$ Department of Radiology, Nara Medical University School of Medicine, Nara, Japan; \\ ${ }^{3}$ Department of Thoracic Surgery, Saiseikai Chuwa Hospital, Nara, Japan; \\ ${ }^{4}$ Shioya Clinic of Internal Medicine, Nara, Japan; \\ ${ }^{5}$ Department of Endoscopy and Ultrasound, Nara Medical University School of Medicine, Nara, Japan
}

\begin{abstract}
Background/Aim: Video-assisted thoracic surgery (VATS) for ipsilateral reoperations is challenging because of the potential for pleural adhesions. Insertion of the initial port can lead to lung injury because of the blinded intrathoracic area. We assessed the usefulness of ultrasonography before VATS to reduce the incidence of lung injury at the time of the initial port insertion during secondary ipsilateral VATS. Patients and Methods: Thirty-three patients who underwent repeat VATS for ipsilateral pulmonary lesions were included. All patients underwent preoperative ultrasonography to assess the possible presence of pleural adhesions using the lung sliding sign. Results: Seven adhesions were found at the VATS ports. Two of these adhesions were not evaluated as pleural adhesions using ultrasonography; however, they were loose. All initial ports were inserted without lung injury. There were no major complications. Conclusion: Preoperative detection of pleural adhesions using ultrasonography can determine the best initial port for secondary ipsilateral VATS.
\end{abstract}

The incidence of secondary thoracic surgery has increased as a result of longer survival times after resection of primary cancers and the widespread use of early detection tools such as computed tomography (1). Furthermore, repeated

This article is freely accessible online.

Correspondence to: Motoaki Yasukawa, Department of Thoracic and Cardiovascular Surgery, Nara Medical University School of Medicine, Kashihara, Nara 634-8522, Japan. Tel: +81 744223051, Fax: +81 744248040, e-mail: myasukawa@naramed-u.ac.jp

Key Words: Pleural adhesion, ultrasonography, secondary thoracotomy. pulmonary metastasectomy offers a chance for long-term survival to patients with prolonged disease-free intervals after initial pulmonary metastasectomy (2). During the last several decades, video-assisted thoracic surgery (VATS) has been validated as the favored technique for almost all thoracic surgical interventions $(3,4)$. However, the use of VATS for ipsilateral reoperations might be challenging because of the potential for pleural adhesions $(5,6)$. Because of secondary intrathoracic changes after the first surgical intervention, pleural adhesions occur frequently in the thoracic cavity and/or chest wall. Several authors (5, 7 ) have reported that almost $80 \%$ of ipsilateral reoperation patients had severe intrathoracic adhesions. The initial insertion port can lead to lung injury because of the blinded intrathoracic area during VATS. However, preoperative transthoracic ultrasonography is useful for detecting pleural adhesions in patients scheduled to undergo thoracotomy (8-11). Therefore, we presumed that preoperative evaluations of pleural adhesions are beneficial before surgical intervention.

This study assessed the usefulness of preoperative ultrasonography to reduce the incidence of lung injury during the initial port insertion during VATS.

\section{Patients and Methods}

Among 547 patients who underwent thoracic surgery at Nara Medical University Hospital from January 2017 to May 2019, 33 who underwent consecutive VATS for reoperations for ipsilateral pulmonary lesions were enrolled. All 33 patients underwent preoperative ultrasonography to assess the possible presence of pleural adhesions.

We used three-port VATS to resect the lung tumors. Placement of the initial port was usually performed in the seventh or eighth intercostal space along the anterior or mid-axillary line. 
An ultrasound study using the LOGIQ E9TM (GE Healthcare, Chicago, IL, USA) ultrasound system was performed within 2 weeks before the scheduled surgery. All patients were examined in the lateral position with the seventh or eighth intercostal space of the midaxillary line undergoing respiration. We evaluated the lung sliding sign, which is the movement of visceral pleural sliding with breathing and is a hallmark of the absence of pleural adhesions. The VATS technique used for reoperation did not differ considerably from that used for routine lung cancer cases, except in the evaluation of adhesions using preoperative ultrasonography. If adhesions were expected on preoperative ultrasonography, the initial port was placed at a location where ultrasonography showed no adhesions.

We checked for adhesions and determined their locations during VATS. Loose adhesions and dense adhesions were considered blunt ablations that either could or could not be removed easily. Prolonged air leakage was defined as an air leak lasting more than 5 days postoperatively. The chest tube duration was measured in days.

The Ethics Review Board of our institute approved the study protocol (No. 1749) and waived the requirement to obtain written informed consent from patients because individual patients were not identified in this retrospective study.

All data were expressed as the mean \pm standard deviation (SD). All statistical analyses were conducted using EZR on R commander version 1.33 (Saitama, Japan) (12).

\section{Results}

Table I shows the clinical characteristics of all patients. Seven adhesions were present at the initial port during VATS. Two adhesions were not detected with preoperative ultrasonography, and the other five adhesions were found using preoperative ultrasonography that was performed for all patients. Among these five cases, we were able to place the initial port while avoiding the adhesions during VATS. Additionally, the two undetected adhesions were removed easily, without lung injury or massive bleeding, because they were loose and could be removed bluntly. Fortunately, there was no conversion from VATS to open thoracotomy. Visceral slide sonography detected the following adhesions: five true-positive adhesions; two false-negative adhesions; zero false-positive adhesions; and 26 true-negative adhesions (sensitivity, 71.4\% [5/7]; specificity, $100.0 \%$ [26/26]; accuracy, 93.9\% [31/33]) (Table II).

Table I shows the outcomes of surgical intervention. The following surgical procedures were performed: pneumonectomy (for one), lobectomy (for four), and wedge (for 28). The mean operative time was 86 minutes ( $\mathrm{SD}, \pm 56$ minutes). The mean intraoperative bleeding volume was $72.4 \mathrm{~g}$ (SD, $\pm 325.1 \mathrm{~g}$; median, $5 \mathrm{~g}$ ). One patient who underwent complete pneumonectomy required blood transfusion intraoperatively. The mean chest tube duration was 1.7 days ( $\mathrm{SD}, \pm 1.7$ days; median, 1 day). Fortunately, there were no complications, such as postoperative bleeding, myocardial infarction, cerebral infarction, arrhythmia, bronchial fistula, or pneumonia for any patients. One patient had prolonged (defined as more than 5 days) air leakage for 10 days.

\section{Discussion}

In this study, evidence is presented that preoperative detection of pleural adhesions using transthoracic ultrasonography could provide safe thoracoscopic access without lung injury and facilitate VATS among ipsilateral secondary thoracic surgery patients, by enabling the placement of the initial port.

Pleural adhesions occur frequently in pulmonary vessels and bronchial and pulmonary surgical stumps in the thoracic cavity and/or chest wall because of secondary intrathoracic changes after the first surgical intervention. Chen et al. (5) have reported that almost $78 \%$ of ipsilateral reoperation patients had severe intrathoracic adhesions. Several authors have reported that surgeons failed to perform complete VATS; there was a $6.5-23 \%$ rate of conversion from complete VATS to open surgery (13-15). Approximately $24 \%$ of conversions were related to intense adhesions and massive intraoperative bleeding (13-15). Therefore, VATS has been rarely used for reoperations for ipsilateral pulmonary lesions.

Several reports of patients who required reoperations for ipsilateral pulmonary lesions have demonstrated that patients in the VATS group had shorter hospital stay and fewer complications than patients in the open thoracotomy. VATS is feasible and safe for selected patients $(5,16,17)$. However, the use of VATS for ipsilateral reoperations might be challenging because of the potential for pleural adhesions. For experienced surgeons, VATS in the presence of dense pleural adhesions might be controversial. Furthermore, for inexperienced surgeons, VATS in the presence of dense pleural adhesions could lead to unexpected massive bleeding and/or lung injury. Specifically, insertion of the initial port can lead to lung injury because of the blinded intrathoracic area during VATS. Preoperative transthoracic ultrasonography is useful for detecting pleural adhesions in patients scheduled to undergo thoracotomy (8-11). We presumed that preoperative evaluations of pleural adhesions are beneficial before surgical interventions.

Preoperative transthoracic ultrasonography was useful for detecting pleural adhesions in patients who had undergone thoracic surgical intervention (8-10). When we could not detect adhesions in patients who did have them, the adhesions were not dense and could be removed easily without injuring the lung. Therefore, before pulmonary resection, preoperative ultrasonography was used to evaluate the feasibility of providing safe thoracoscopic access without lung injury and/or unexpected massive bleeding, thereby facilitating VATS (11). The lung sliding sign can discriminate between dense and non-dense adhesions. If there were dense adhesions, then the lung would not slide with breathing. If there were no adhesions or if there were loose adhesions, then the lung would slide with breathing. Regarding the safety of VATS, especially when adhesions are present, it is important to not discriminate between adhesions and no 
Table I. Clinical characteristics of all patients.

\begin{tabular}{lc}
\hline & All patients $(\mathrm{n}=33)$ \\
\hline Age (years) & $67.1 \pm 13.8$ \\
Gender, $\mathrm{n}(\%)$ & \\
$\quad$ Male & $24(72.7)$ \\
Female & $9(27.3)$ \\
Procedure, $\mathrm{n}(\%)$ & \\
$\quad$ Pneumonectomy & $1(3.0)$ \\
Lobectomy & $4(12.1)$ \\
Wedge resection & $28(84.8)$ \\
Thoracic adhesions observed, $\mathrm{n}(\%)$ & $2(6.1)$ \\
Dense & $5(15.2)$ \\
Loose & $26(78.8)$ \\
None & $86 \pm 56$ \\
Length of surgery (min) & $72.5 \pm 325.1$ \\
Intraoperative bleeding (g) & $1.7 \pm 1.7$ \\
Chest tube duration (days) & $1(3.0)$ \\
Postoperative complications, $\mathrm{n}(\%)$ & prolonged air leakage \\
&
\end{tabular}

adhesions; instead, one should distinguish between simple cases and difficult cases and between high potential and low potential for complications.

During this study, operative time, intraoperative bleeding, and chest tube duration were acceptable for patients who underwent ipsilateral secondary thoracotomy. Furthermore, there were no conversions from VATS to open thoracotomy. Therefore, using ultrasonography might be a helpful method of reducing complications during VATS for ipsilateral secondary lung resection patients. Moreover, use of preoperative ultrasonography could decrease factors of prolonged postoperative air leakage due to pleural adhesions.

Preoperative ultrasonography was used to determine the existence of adhesions in lung neoplasm patients undergoing reoperation. Unfortunately, excepted port site adhesions were not examined because it is difficult to detect adhesions near the apex area with ultrasonography. Especially for ipsilateral secondary VATS, insertion of the initial port can be the most crucial point of leading to lung injury because of the blinded intrathoracic area. If adhesions were visible, adhesions can be removed carefully during VATS, then pulmonary resection can be performed safely, without lung injury and massive bleeding.

In the near future, uniportal VATS will be more widespread than conventional multiport VATS $(3,4)$. Preoperative evaluation of port site and placing the port while avoiding the adhesions would be of significant importance in the uniportal VATS era. Ultrasonography might become a more important examination for clinicians. Ultrasonography is a simple non-invasive procedure that provides real-time and immediate results. Furthermore, ultrasonography offers other advantages; free from radiation hazards, portable, and relatively cost-effective (18). Because
Table II. Results following lung slide sign identification using ultrasonography to detect pleural adhesions.

\begin{tabular}{lcc}
\hline Adhesions predicted & Adhesions observed & Adhesions observed \\
\cline { 2 - 3 } & Yes & No \\
\hline Yes & 5 & 0 \\
No & 2 & 26 \\
\hline
\end{tabular}

Adhesions were found at the initial port in all patients $(n=33)$.

ultrasonography is easy to learn and use, portable, and accurate when examining the pleural space, it has allowed for safer pleural procedures, including that of VATS.

This study has certain limitations. First, it was a singleinstitute study with a small sample size. Therefore, it is necessary to perform additional investigations involving a large multi-institutional cohort to validate these findings. Second, as mentioned, the expected port site adhesions were not examined during this study because it is difficult to detect adhesions near the apex site with ultrasonography.

In conclusion, preoperative detection of pleural adhesions using transthoracic ultrasonography was useful for ipsilateral secondary pulmonary resection patients undergoing VATS. Using preoperative ultrasonography can improve the safety and feasibility of placing the initial port in VATS.

\section{Conflicts of Interest}

The Authors declare that they have no conflicts of interest in regard to this study.

\section{Authors' Contributions}

MY is the corresponding author and wrote the manuscript. RT, NM, and TH performed the ultrasonographic diagnosis. TK, NS, TT, and MY participated in the surgery and postoperative management. TT, JT, NH, and ST supervised the writing of the manuscript. All Authors read and approved the final manuscript.

\section{References}

1 Bae MK, Byun CS, Lee CY, Lee JG, Park IK, Kim DJ and Chung KY: The role of surgical treatment in second primary lung cancer. Ann Thorac Surg 92: 256-263, 2011. PMID: 21600562. DOI: 10.1016/j.athoracsur.2011.02.034

2 Chen F, Sakai H, Miyahara R, Bando T, Okubo K and Date H: Repeat resection of pulmonary metastasis is beneficial for patients with colorectal carcinoma. World J Surg 34: 2373-2378, 2010. PMID: 20582543. DOI: 10.1007/s00268-010-0695-x

3 Reinersman JM, Passera E and Rocco G: Overview of uniportal video-assisted thoracic surgery (VATS): past and present. Ann Cardiothorac Surg 5: 112-117, 2017. PMID: 27134837. DOI: 10.21037/acs.2016.03.08 
4 Mineo TC and Ambrogi V: A glance at the history of uniportal video-assisted thoracic surgery. J Vis Surg 3: 157, 2017. PMID: 29302433. DOI: 10.21037/jovs.2017.10.11

5 Chen D, Mao R, Kadeer X, Sun W, Zhu E, Peg Q and Chen C: Video-assisted thoracic surgery is an optimal alternative to conventional thoracotomy for reoperations for ipsilateral pulmonary lesions. Thoracic Cancer 9: 1421-1428, 2018. PMID: 30152592. DOI: $10.1111 / 1759-7714.12854$

6 Dziedzic D and Orlowski T: The role of VATS in lung cancer surgery: Current status and prospects for development. Minim Invasive Surg 2015: 938430, 2015. PMID: 26294970. DOI: $10.1155 / 2015 / 938430$

7 Holbek BL, Petersen RH and Hansen HJ: Is it safe to perform completion lobectomy after diagnostic wedge resection using video-assisted thoracoscopic surgery? Gen Thorac Cardiovasc Surg 64: 203-208, 2016. PMID: 26914890. DOI: 10.1007/ s11748-016-0633-4

8 Sasaki M, Kawabe M, Hirai S, Yamada N, Morioka K, Ihaya A and Tanaka K: Preoperative detection of pleural adhesions by chest ultrasonography. Ann Thorac Surg 80: 439-442, 2005. PMID: 16039181. DOI: 10.1016/j.athoracsur.2005.03.021

9 Wei B, Wang T, Jiang F and Wang H: Use of transthoracic ultrasound to predict pleural adhesions: a prospective blinded study. Thorac Cardiovasc Surg 60: 101-104, 2012. PMID: 21442577. DOI: $10.1055 / \mathrm{s}-0030-1270760$

10 Cassanelli N, Caroli G, Dolci G, Dell'Amore A, Luciano G, Bini A and Stella F: Accuracy of transthoracic ultrasound for the detection of pleural adhesions. Eur J Cardiothorac Surg 42: 813818, 2012. PMID: 22518039. DOI: 10.1093/ejcts/ezs144

11 Yasukawa M, Taiji R, Marugami N, Kawaguchi T, Kawai N,Sawabata N, Toko T, Takahama J, Hamazaki N, Hirai T, and Taniguchi S: Ultrasonography for detecting adhesions: Aspirin continuation for lung resection patients. In Vivo 33: 973-978, 2019. PMID: 31028224. DOI: 10:21873/invivo.11566

12 Kanda Y: Investigation of the freely available easy-to-use software 'EZR' for medical statistics. Bone Marrow Transplant 48: 452-458, 2013. PMID: 23208313. DOI: 10.1038/bmt. 2012.244
13 Samson P, Guitron J, Reed MF, Hanseman DJ and Starnes SL: Predictors of conversion to thoracotomy for video-assisted thoracoscopic lobectomy: A retrospective analysis and the influence of computed tomography -based calcification assessment. J Thorac Cardiovasc Surg 145: 1512-1518, 2013. PMID: 22698554. DOI: 10.1016/j.jtcvs.2012.05.028

14 Puri V, Patel A, Majumder K, Bell JM, Crabtree TD, Krupnick AS, Kreisel D, Broderick SR, Patterson GA and Meyers BF: Intraoperative conversion from video-assisted thoracoscopic surgery lobectomy to open thoracotomy: A study of causes and implications. J Thorac Cardiovasc Surg 149: 55-61, 2015. PMID: 25439768. DOI: 10.1016/j.jtcvs.2014.08.074

15 Augustin F, Maier HT, Weissenbacher A, Ng C, Lucciarini P, Öfner D, Ulmer $\mathrm{H}$ and Schmid T: Causes, predictors and consequences of conversion from VATS to open lung lobectomy. Surg Endosc 30: 2415-2421, 2016. PMID: 26335073. DOI: 10.1007/s00464-015-4495-3

16 Yang J, Xia Y, Yang Y, Ni ZZ, He WX, Wang HF, Xu XX, Yang YL, Fei K and Jiang GN: Risk factors for major adverse events of video-assisted thoracic surgery lobectomy for lung cancer. Int J Med Sci 11: 863-869, 2014. PMID: 25013365. DOI: 10.7150/ ijms. 8912

17 Sun W, Zhang L, Li Z, Chen D, Jiang G, Hu J and Cahen C: Feasibility investigation of ipsilateral reoperations by thoracoscopy for major lung resection. Thorac Cardiovasc Surg 28, 2019. PMID: 30921828. DOI: 10.1055/s-0039-1683372

18 Rumende CM: The role of ultrasonography in the management of lung and pleural diseases. Acta Med Indones 44: 175-183, 2012. PMID: 22745151. 